\title{
STM Study of Mixed Alkanethiol/Biphenylthiol Self-Assembled Monolayers on Au(111)
}

\author{
Björn Lüssem, ${ }^{* \dagger}$ Lars Müller-Meskamp, ${ }^{\dagger}$ Silvia Karthäuser, ${ }^{\dagger}$ Rainer Waser, ${ }^{\dagger, \ddagger}$ \\ Melanie Homberger, ${ }^{\S}$ and Ulrich Simon ${ }^{\S}$
}

Institute for Solid State Research and Center of Nanoelectronic Systems for Information Technology, Research Centre Jülich GmbH, 52425 Jülich, Germany, Institut für Werkstoffe der Elektrotechnik 2, RWTH Aachen, Sommerfeldstrasse 24, 52074 Aachen, Germany, and Institute for Inorganic Chemistry, RWTH Aachen, Landoltweg1, 52074 Aachen, Germany

Received October 17, 2005. In Final Form: January 12, 2006

\begin{abstract}
A method is presented for depositing mixed self-assembled monolayers (SAMs) of dodecanethiol (C12) and 4'methyl-1,1'-biphenyl-4-butane $\left(\mathrm{H}_{3} \mathrm{C}-\mathrm{C}_{6} \mathrm{H}_{4}-\mathrm{C}_{6} \mathrm{H}_{4}-\left(\mathrm{CH}_{2}\right)_{4}-\mathrm{SH}, \mathrm{BP} 4\right)$ by insertion of BP4 into a closely packed SAM of dodecanethiol on $\mathrm{Au}(111)$. Insertion takes place at defect sites such as domain boundaries or etch pits in the gold surface that are characteristic of $\mathrm{C} 12$ monolayers on gold. With a lower probability, insertion also occurs beside defect sites inside dodecanethiol domains. Insertion at defect sites results in domains of BP4, whereas insertion into C12 domains leads to isolated BP4 molecules. The isolated BP4 molecules are shown not to move at room temperature. By comparing the apparent height of the isolated BP4 molecules and BP4 domains, it is proposed that the isolated molecules have the same conformation as in the full-coverage phase. A simple two-layer model is proposed to characterize the current transport through BP4. The decay constant $\beta$ for the phenylene groups is deduced from the apparent STM heights of the inserted BP4 islands compared to the STM heights of the C12 closely packed monolayers.
\end{abstract}

\section{Introduction}

Thiol-based self-assembled monolayers (SAMs) are an effective tool to use in tailoring the surface properties of metals..$^{1,2}$ The applications of SAMs range from wetting control, ${ }^{3-6}$ corrosion inhibition, ${ }^{7}$ protein adsorption, ${ }^{8}$ and lateral structuring ${ }^{9}$ to molecular electronics. ${ }^{10}$ For most of these applications, SAMs that consist of more than one component (mixed SAMs) are used because these SAMs exhibit more degrees of freedom (i.e., the surface properties can be adjusted by the ratio of the different constituents in the film). This approach is especially promising for molecular electronics. Weiss et al. were the first to characterize electronic transport properties of single molecules via an STM setup by using mixed SAMs. A molecular wire candidate, a conjugated organic molecule, was diluted in a SAM of docecanethiol. ${ }^{10}$ Thereby, the molecular wire was indeed shown to be highly conductive compared to the alkanethiols.

There are several ways to prepare mixed SAMs. One method is to assemble the film from a mixture of different substances (coadsorption). Various mixed SAMs have been realized using this approach: alkanethiols with different chain lengths ${ }^{11,12}$ and

* To whom correspondence should be addressed. E-mail: b.luessem@ fz-juelich.de.

$\dagger$ Institute for Solid State Research and Center of Nanoelectronic Systems for Information Technology.

$\stackrel{\doteqdot}{\doteqdot}$ Institut für Werkstoffe der Elektrotechnik 2, RWTH Aachen.

$\S$ Institute for Inorganic Chemistry, RWTH Aachen.

(1) Schreiber, F. Prog. Surf. Sci. 2000, 65, 151.

(2) Love, J. C.; Estroff, L. A.; Kriebel, J. K.; Nuzzo, R. G.; Whitesides, G. M. Chem. Rev. 2005, 105, 1103.

(3) Bain, C. D.; Whitesides, G. M. J. Am. Chem. Soc. 1988, 110, 6560

(4) Arte, S. V.; Liedberg, B.; Allara, D. L. Langmuir 1995, 11, 3882

(5) Morgenthaler, S.; Lee, S.; Zürcher, S.; Spencer, N. D. Langmuir 2003, 19, 10459

(6) Yamada, R.; Tada, H. Langmuir 2005, 21, 4254

(7) Scherer, J.; Vogt, M. R.; Magnussen, O. M.; Behm, R. J. Langmuir 1997, 13,7045 .

(8) Prime, K. L.; Whitesides, G. M. Science 1991, 252, 1164.

(9) Kumar, A.; Whitesides, G. M. Appl. Phys. Lett. 1993, 63, 2002.

(10) Bumm, L. A.; Arnold, J. J.; Cygan, M. T.; Dunbar, T. D.; Burgin, T. P.; Jones, L., II; Allara, D. L.; Tour, J. M.; Weiss, P. S. Science 1996, 271, 1705

(11) Chen, S.; Li, L.; Boozer, C. L.; Jiang, S. Langmuir 2000, 16, 9287. different terminal groups ${ }^{12-14}$ or decanethiol and an amidecontaining alkanethiol of similar length. ${ }^{15}$ Bain et al. ${ }^{12}$ showed that the composition of the monolayer does not fully correspond to the composition of the solution and that longer chains are preferentially adsorbed, which points toward thermodynamic control of the adsorption process. This was confirmed later by a theoretical study. ${ }^{16}$

Another method is the adsorption of asymmetric disulfides. ${ }^{13-22}$ By this method, different mixed SAMs have been prepared (e.g., mixed SAMs of alkanethiols having different tail groups ${ }^{13,17}$ and chain lengths, ${ }^{23}$ symmetrical and asymmetrical alkyl and perfluoroalkyl disulfides, ${ }^{18-20}$ asymmetrical diethylalkanoat disulfides, ${ }^{21}$ and diacetylene disulfide..$^{22}$

In contrast to the two previous methods, which always consist of a single deposition process, a two-step deposition process is mainly used for isolating electrically active molecules in a host matrix. ${ }^{10,24-31}$ For this method, a preassembled, closely packed SAM (mostly consisting of insulating alkanethiols) is dipped in

(12) Bain, C. D.; Whitesides, G. M. J. Am. Chem. Soc. 1989, 111, 7164.

(13) Takami, T.; Delamarche, E.; Michel, B.; Gerber, C.; Wolf, H.; Ringsdorf, H. Langmuir 1995, 11, 3876.

(14) Li, L.; Cheng, S.; Jiang, S. Langmuir 2003, 19, 3266.

(15) Smith, R. K.; Reed, S. M.; Lewis, P. A.; Monnell, J. D.; Clegg, R. S.; Kelly, K. F.; Bumm, L. A.; Hutchison, J. E.; Weiss, P. S. J. Phys. Chem. B 2001, 105,1119

(16) Shevade, A. V.; Zhou, J.; Zin, M. T.; Jiang, S. Langmuir 2001, 17, 7566. 2975.

(18) Jaschke, M.; Schönherr, H.; Wolf, H.; Butt, H.-J.; Bamberg, E.; Besocke, M. K.; Ringsdorf, H. J. Phys. Chem. 1996, 100, 2290.

(19) Schönherr, H.; Ringsdorf, H. Langmuir 1996, 12, 3891.

(20) Schönherr, H.; Ringsdorf, H.; Jaschke, M.; Butt, H.-J.; Bamberg, E.; Allinson, H.; Evans, S. D. Langmuir 1996, 12, 3898

(21) Nelles, G.; Schönherr, H.; Vancso, G. J.; Butt, H.-J. Appl. Phys. A 1998 66, S1261.

(22) Nelles, G.; Schönherr, H.; Jaschke, M.; Wolf, H.; Schaub, M.; Küther, J.; Tremel, W.; Bamberg, E.; Ringsdorf, H.; Butt, H.-J. Langmuir 1998, 14, 808.

(23) Heister, K.; Allara, D. L.; Bahnck, K.; Frey, S.; Zharnikov, M.; Grunze, M. Langmuir 1999, 15, 5440.

(24) Cygan, M. T.; Dunbar, T. D.; Arnold, J. J.; Bumm, L. A.; Shedlock, N. F.; Burgin, T. P.; Jones, L., II; Allara, D. L.; Tour, J. M.; Weiss, P. S. J. Am. Chem Soc. 1998, 120, 2721. 
a second step into a solution of the electronically active molecules. In this second step, molecules of the host matrix are exchanged by the electrically active molecules. It was shown that this process mainly takes place at defects in the host matrix. ${ }^{10,24,25}$ Studies on the insertion of linear conjugated molecules have shown that the number of molecules incorporated into the monolayer is a function of immersion time. ${ }^{25}$ The rate-determining step is the replacement of host molecules by molecules from the second molecular solution. ${ }^{32}$

The use of the two-step deposition process is not limited to embedding electrically interesting molecules into an insulating SAM but is rather a more general tool for the construction of molecular devices in a bottom-up approach. For example, complex dendrimer molecules ${ }^{32,33}$ and nanosize molecular cages ${ }^{34}$ have been inserted into a preassembled SAM.

Depending on the preparation technique and the molecular species used, the monolayers are either homogeneously mixed or the different molecules segregate into separate domains. For some applications, uniformly mixed monolayers are preferred, so a kinetically trapped method to prepare truly molecular-scale mixed SAMs has been developed. ${ }^{11}$ Similarly, the deposition of mixed monolayers from asymmetrical disulfides results in uniform mixed layers at room temperature..$^{13,17,19}$ At higher temperatures $\left(100^{\circ} \mathrm{C}\right)$, Ishida et al. observed phase separation in a SAM formed by an asymmetric disulfide with hydrocarbon and fluorocarbon chains, ${ }^{35}$ whereas Schönherr et al. observed no phase segregation in mixed alkyl fluoroalkyl disulfides. ${ }^{20}$

In most of the molecular electronic studies that measure the electronic transport along a molecular wire, the electronically active molecules are also rather isolated in the host matrix than surrounded by a segregated domain of the same molecule. ${ }^{10,24-26,28,29}$ In some of these studies, the apparent height in the STM images of the molecules above the host matrix is related to the current transport properties. This is possible because the STM height does not solely reflect the physical height of the molecule but also comprises the transconductance of the molecule.

However, the exact conformation of embedded single molecules is mainly unknown, and there exist only a few studies that have addressed this question. ${ }^{25}$ Furthermore, the molecules are inserted at a variety of defects in the host matrix, which may affect the electrical properties of the molecules. This lack of information makes an exact interpretation of the current transport along these molecules difficult. In contrast to embedded single molecules, the structure and conformation of molecules in closely packed SAMs are studied in more detail ${ }^{1,36}$ These structures should prevail in phase-segregated mixed SAMs if the domains

(25) Dunbar, T. D.; Cygan, M. T.; Bumm, L. A.; McCarty, G. S.; Burgin, T. P.; Reinerth, W. A.; Jones, L., II; Jackiw, J. J.; Tour, J. M.; Weiss, P. S.; Allara, D. L. J. Phys. Chem. B 2000, 104, 4880.

(26) Wakamatsu, S.; Akiba, U.; Fujihira, M. Colloids Surf., A 2002, 198, 785.

(27) Nakasa, A.; Akiba, U.; Fujihira, M. Appl. Surf. Sci. 2000, 157, 326.

(28) Moth-Poulsen, K.; Patrone, L.; Stuhr-Hansen, N.; Christensen, J. B.; Bourgoin, J.-P.; Bjørnholm, T. Nano Lett. 2005, 5, 783.

(29) Szuchmacher Blum, A.; Ren, T.; Parish, D. A.; Trammell, S. A.; Moore, M. H.; Kushmerick, J. G.; Xu, G.-L.; Deschamps, J. R.; Pollack, S. K.; Sashidhar, R. J. Am. Chem. Soc. 2005, 127, 10010.

(30) Ishida, T.; Mizutani, W.; Choi, N.; Akiba, U.; Fujihira, M.; Tokumoto, H. J. Phys. Chem. B. 2000, 104, 11680 .

(31) Ishida, T.; Mizutani, W.; Tokumoto, H.; Choi, N.; Akiba, U.; Fujihira, M. J. Vac. Sci. Technol., A 2000, 18, 1437.

(32) Figgeri, A.; Schönherr, H.; van Manen, H.-J.; Huisman, B.-H.; Vansco, G. J.; Huskens, J.; van Veggel, F. C. J. M.; Reinhoudt, D. N. Langmuir 2000, 16,7757 .

(33) Huisman, B.-H.; Schönherr, H.; Huck, W. T. S.; Friggeri, A.; van Manen, H.-J.; Menozzi, E.; Vancso, G. J.; van Veggel, F. C. J. M.; Reinhoudt, D. N. Angew. Chem., Int. Ed. 1999, 38, 2248.

(34) Menozzi, E.; Pinalli, R.; Speets, E. A.; Ravoo, B. J.; Dalcanale, E.; Reinhoudt, D. N. Chem.-Eur. J. 2004, 10, 2199.

(35) Ishida, T.; Yamamoto, S.; Mizutani, W.; Motomatsu, M.; Tokumoto, H.; Hokari, H.; Azehara, H.; Fujihira, M. Langmuir 1997, 13, 3261. are large enough so that the STM height difference between separate domains in a mixed SAM can be interpreted in terms of the transconductance of different molecules without having uncertainties in the measurements on embedded single molecules. $^{37}$

Probably the most studied SAMs are SAMs of alkanethiols on gold. ${ }^{1}$ These SAMs are known to form a $(\sqrt{ } 3 \times \sqrt{ } 3)$ R30 overlayer on the (111) gold surface, which is further structured by a $c(4 \times 2)$ superstructure. ${ }^{38-43}$ The superstructure is characterized by the systematic arrangement of molecules showing distinct height differences in STM images. Vertically, the alkane chain is tilted $\sim 30^{\circ}$ with respect to the surface normal. ${ }^{44}$

In comparison to alkanethiols, which are highly insulating, self-assembled monolayers of biphenylthiols $\left(\mathrm{H}_{3} \mathrm{C}-\mathrm{C}_{6} \mathrm{H}_{4}-\right.$ $\left.\mathrm{C}_{6} \mathrm{H}_{4}-\left(\mathrm{CH}_{2}\right)_{n}-\mathrm{SH}, \mathrm{BPn}\right)$ are supposed to show more interesting electronic properties. Because of the conjugation in the upper part of the molecule, the transconductance of the molecules should be higher than that of alkanethiols. The structure of biphenylthiols on (111)-oriented gold has been examined by Azzam et al. In particular, they show that BP4 adopts a $(5 \sqrt{ } 3 \times 3)$ structure. ${ }^{45}$ The orientation of the molecules perpendicular to the substrate has been examined by Rong et al. ${ }^{36}$ It is shown that, for an even number of carbon atoms in the alkyl chain, the alkanethiol group adopts a rather upright orientation whereas the biphenyl group is tilted by approximately $45^{\circ}$ out of the surface normal (Figure 7).

Biphenylthiols are ideal substances to insert into an alkanethiol host matrix. Compared to the conjugated molecules used by Bumm et al., ${ }^{10}$ BP4 can better adapt to the alkanethiol lattice because of the higher flexibility of the alkane chain. This can be particularly important in the rate-determining exchange of alkanethiols by biphenylthiols. However, alkanethiols and biphenylthiols show sufficient differences in their molecular structure to cause phase segregation. ${ }^{16}$ As described above, the height difference of these domains measured by STM can be interpreted as a combination of the physical height difference and the difference in transconductance of the molecules. Compared to the molecules used by Ishida et al., ${ }^{30,31} \mathrm{BP} 4$ and C12 have the same top and bottom groups, which simplifies the interpretation. Therefore, we prepared mixed SAMs consisting of isolating dodecanethiol (C12) and BP4 by a two-step process with the aim of characterizing the current transport through biphenylthiols.

\section{Experimental Details}

C12 and absolute ethanol are purchased from Aldrich and used as received. Analytically pure BP4 is synthesized according to Buck et al. via a Grignard $\mathrm{C}-\mathrm{C}$ coupling reaction of the corresponding phenyl- or alkylbromide followed by the conversion of the bromide to the thiol via thiourea. ${ }^{36}$ Characterization of the BP4 is done via NMR, mass spectrometry, and elemental analysis.

(36) Rong, H.-T.; Frey, S.; Yang, Y.-J.; Zharnikov, M.; Buck, M.; Wühn, M.; Wöll, C.; Helmchen, G. Langmuir 2001, 17, 1582.

(37) Bumm, L. A.; Arnold, J. J.; Dunbar, T. D.; Allara, D. L.; Weiss, P. S J. Phys. Chem. B 1999, 103, 8122.

(38) Camillone, N.; C. E. Chidsey, D.; Liu, G.; Scoles, G. J. Chem. Phys. 1993, 98, 3503.

(39) Poirier, G. E.; Tarlov, M. J. Langmuir 1994, 10, 2853.

(40) Delamarche, E.; Michel, B.; Gerber, C.; Anselmetti, D.; Güntherodt, H. J.; Wolf, H.; Ringsdorf, H. Langmuir 1994, 10, 2869.

(41) Zhang, L.; Goddard, W. A., III; Jiang, S. J. Chem. Phys. 2002, 117, 7342

(42) Lüssem, B.; Müller-Meskamp, L.; Karthäuser, S.; Waser, R. Langmuir $\mathbf{2 0 0 5}, 21,5256$.

(43) Müller-Meskamp, L.; Lüssem, B.; Karthäuser, S.; Waser, R. J. Phys. Chem. B 2005, 109, 11424.

(44) Fenter, P.; Eberhardt, A.; Liang, K. S.; Eisenberger, P. J. Chem. Phys. 1997, 106, 1600 .

(45) Azzam, W.; Cyganik, P.; Witte, G.; Buck, M.; Wöll, C. Langmuir 2003, 19,8262 . 
(a)

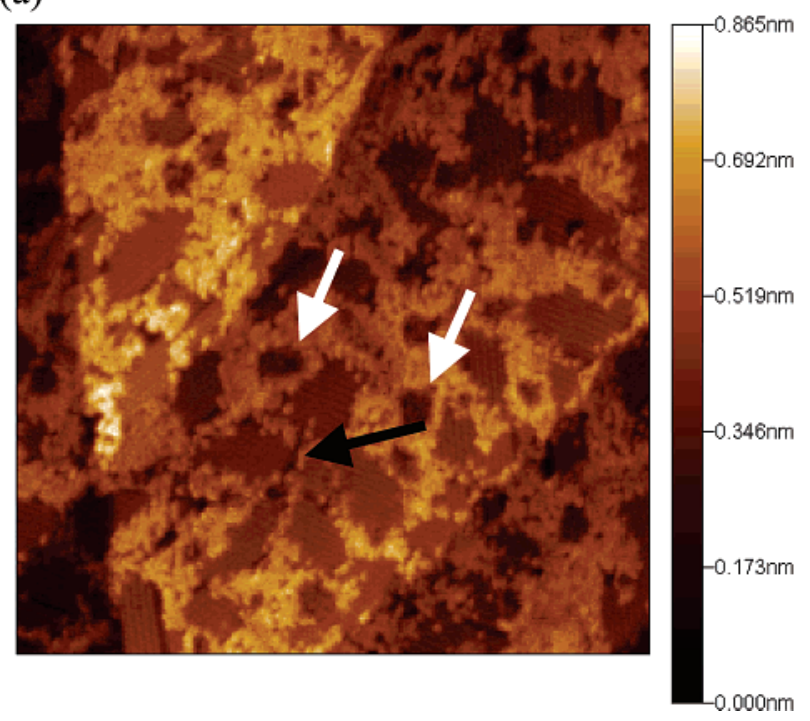

(b)

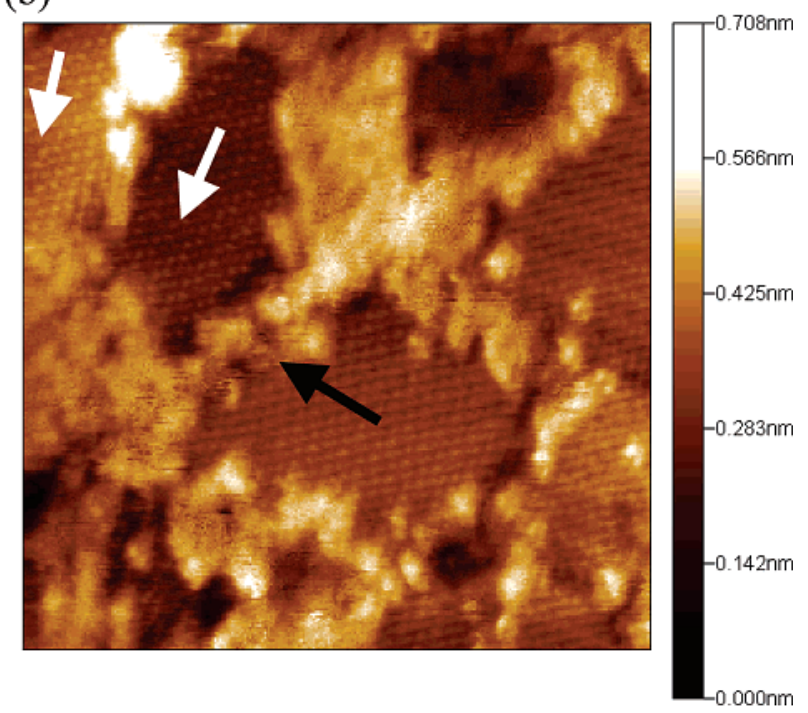

Figure 1. STM scans $\left\{\right.$ (a) $100 \times 100 \mathrm{~nm}^{2}$ and (b) $\left.30 \times 30 \mathrm{~nm}^{2}\right\}$ of a C12/BP4 mixed monolayer. The film shows separate BP4 and C12 domains. Higher appearing domains consist of BP4. In the higher-resolution scan, the $\mathrm{c}(4 \times 2)$ structure of $\mathrm{C} 12$ is visible (white arrows in b). Black arrows mark the nucleation of BP4 domains at C12 domain boundaries, and the white arrows in part a mark the nucleation of BP4 domains at holes in the gold surface.

(a)

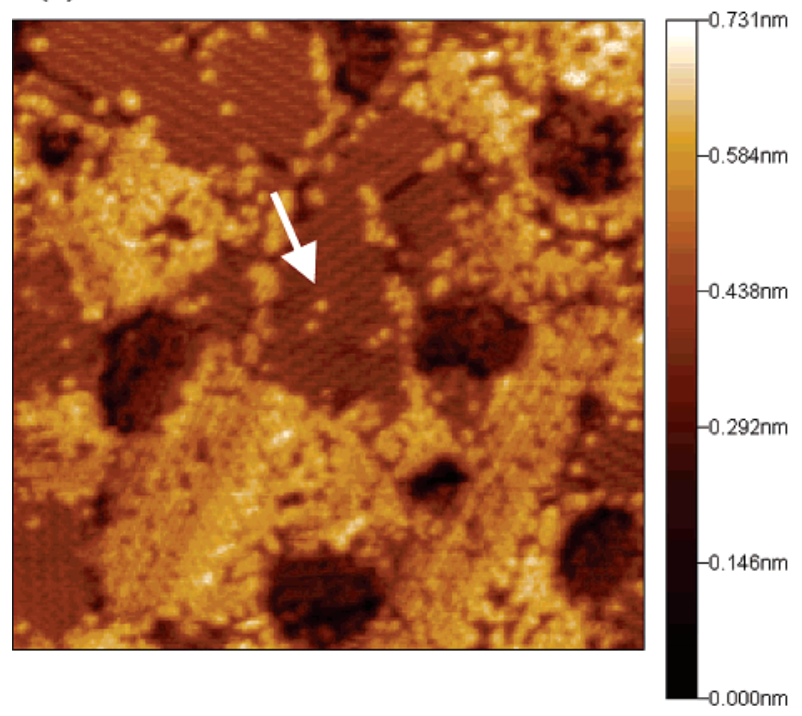

(b)

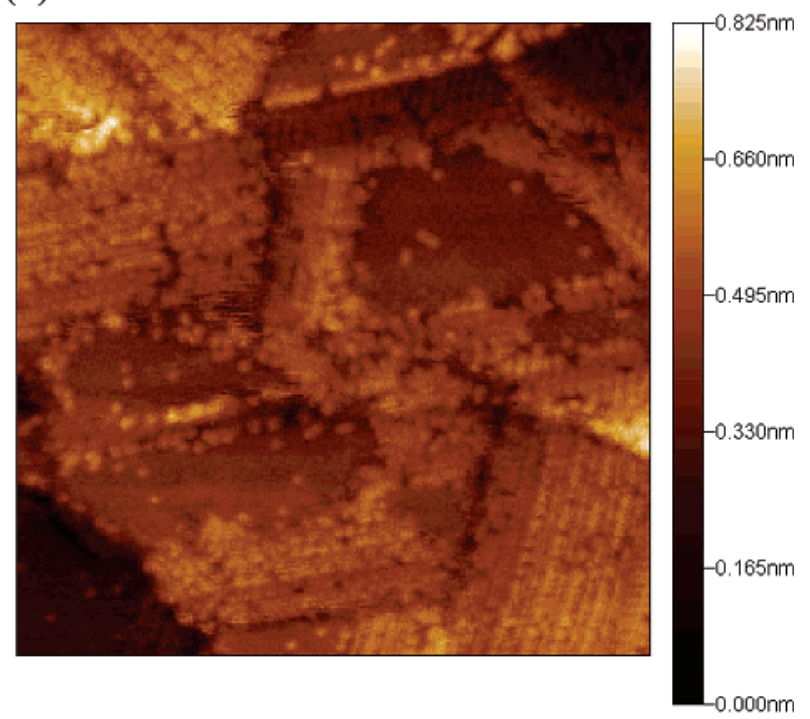

Figure 2. Scans $\left(50 \times 50 \mathrm{~nm}^{2}\right)$ of a mixed C12/BP4 monolayer annealed at $64{ }^{\circ} \mathrm{C}$ for (a) 90 and (b) 225 min. An increase in structural order of the BP4 domains is visible in the texture of these domains. The white arrow in part a marks a BP4 molecule inserted into the middle of a C12 domain.

Gold films are deposited onto mica by a standard procedure that results in (111)-oriented gold films with large terraces. ${ }^{46} \mathrm{C} 12$ is deposited by dipping the gold film into a $1 \mathrm{mM}$ ethanolic solution of dodecanethiol overnight. The insertion of BP4 is accomplished by immersing the closely packed SAM of C12 for 20 min into a 100 $\mu \mathrm{M}$ solution of $\mathrm{BP} 4$ in ethanol at $70{ }^{\circ} \mathrm{C}$. This deposition procedure results in phase-segregated islands of $\mathrm{BP} 4$ and $\mathrm{C} 12$, shorter immersion times (10 $\mathrm{min}$ ), and lower temperatures (RT) to yield single embedded molecules or bundles with a small number of molecules.

The films are characterized by ultrahigh vacuum scanning tunneling microscopy (UHV-STM) (JEOL JSPM 4500) at a base pressure of $3 \times 10^{-10}$ mbar. Homemade tungsten STM tips are used. All STM scans are taken in constant current mode at a tunneling voltage of $2 \mathrm{~V}$ and a tunneling current of $50 \mathrm{pA}$.

(46) Lüssem, B.; Karthäuser, S.; Haselier, H.; Waser, R. Appl. Surf. Sci. 2005, 249,197

\section{Results and Discussions}

In Figure 1, STM images of a mixed SAM consisting of C12 and BP4 are shown. In the large-area scan (Figure 1a), several gold terraces are seen that are covered by separate domains of BP4 and C12. Domains of BP4 and C12 can be distinguished by the high-resolution scan shown in Figure 1b. In this scan, the lower appearing domains are molecularly resolved, and especially in the domains in the upper left corner the $c(4 \times 2)$ structure of alkanethiols on gold can be seen (white arrows). Therefore, the lower appearing domains can be assigned to C12. Additionally, in the large-area scan deep pits corresponding to monatomic gold steps are seen, which are characteristic of SAMs of thiols on gold (white arrows in Figure 1a). ${ }^{47}$ These holes are mostly surrounded by domains of BP4. Similarly, at domain boundaries of different $\mathrm{C} 12$ domains, islands of BP4 start to grow (black

(47) Poirier, G. E. Langmuir 1997, 13, 2019. 


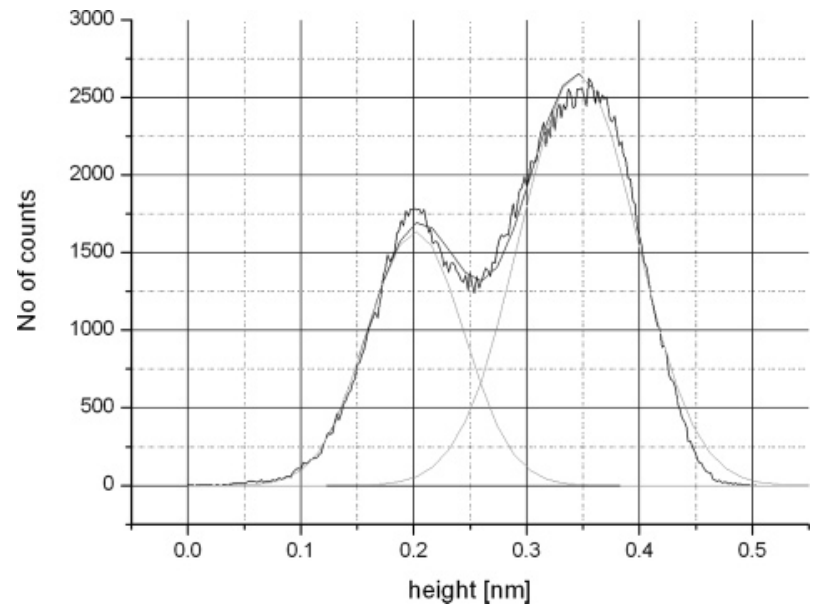

Figure 3. Height distribution of an STM scan of a mixed C12/BP4 monolayer. Two peaks that correspond to the different heights of the different domains are visible. The distance from the center of these peaks is taken as the mean height difference of the $\mathrm{C} 12$ and BP4 domains.

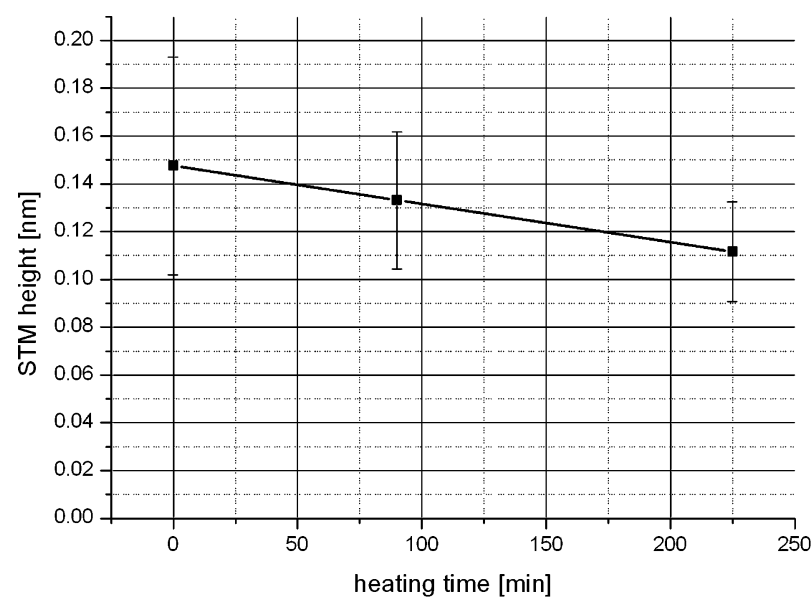

Figure 4. Height difference of BP4 and $\mathrm{C} 12$ domains depending on annealing time.

arrows in Figure 1a and b), indicating that domain boundaries of the host matrix and pits in the gold surface act as nucleation points for BP4 domains, confirming the results of Cygan and
Weiss et al. for other electronically active molecules. ${ }^{24}$ However, most BP4 domains in this and later measurements grow around pits so that the exchange of $\mathrm{C} 12$ molecules by $\mathrm{BP} 4$ molecules seems to be more effective at these defect sites.

In contrast to domains of $\mathrm{C} 12, \mathrm{BP} 4$ domains exhibit a rather amorphous texture. To increase the order in these films, we have annealed the samples at $64{ }^{\circ} \mathrm{C}$ in a vacuum for several hours. An STM scan of the resulting film after $1.5 \mathrm{~h}$ is shown in Figure $2 \mathrm{a}$ and after $3 \mathrm{~h} 45 \mathrm{~min}$ in Figure 2b. The appearance of the BP4 islands changes from amorphous and cloudy (Figure 1) to a more regular shape with sharp boundaries between adjacent $\mathrm{C} 12$ and BP4 domains. However, as a consequence of the different tunneling conditions above a BP4 and a $\mathrm{C} 12$ domain, it was not possible to resolve the $\mathrm{BP} 4$ domains molecularly. The tunneling impedance was chosen to be as high as possible $(2 \mathrm{~V}, 50 \mathrm{pA})$ to ensure that in any part of the mixed SAM the tip is not penetrating the molecular monolayer. Whereas for the $\mathrm{C} 12$ domains this tunneling impedance yields a high resolution, BP4 films are best scanned with a lower impedance $(0.5 \mathrm{~V}, \sim 150$ $\mathrm{pA}^{45}$ ). Such a low impedance would cause the STM tip to push into the $\mathrm{C} 12$ domains, thereby limiting the resolution and, most importantly, inhibiting the interpretation of the height differences in terms of different molecular conductivities as given in the last part of this article.

Although the precise molecular structure of the BP4 domains cannot be deduced, the increase in order can be quantified by a decrease in the roughness of the film due to annealing. Whereas the as-deposited film exhibits a roughness (rms value) of 0.12 $\mathrm{nm}$ on an area of $50 \times 50 \mathrm{~nm}^{2}$, this roughness slightly decreases to $0.102 \mathrm{~nm}$ for the annealed films.

The increase in order in the film by an additional annealing step suggests that there is some rearrangement in the BP4 films, which should result in a change in the height difference of the BP4 and C12 domains.

To survey the height differences before and after annealing, the height difference is evaluated as follows. From STM scans such as those shown in Figures 1 and 2, the histogram of the height of each image pixel is taken (Figure 3). These histograms display two distinct peaks: the lower one for the height of the $\mathrm{C} 12$ domains and the higher one for the BP4 domains. Both peaks are fitted to a Gaussian distribution, and the difference in the center positions of the peaks is taken as the mean height
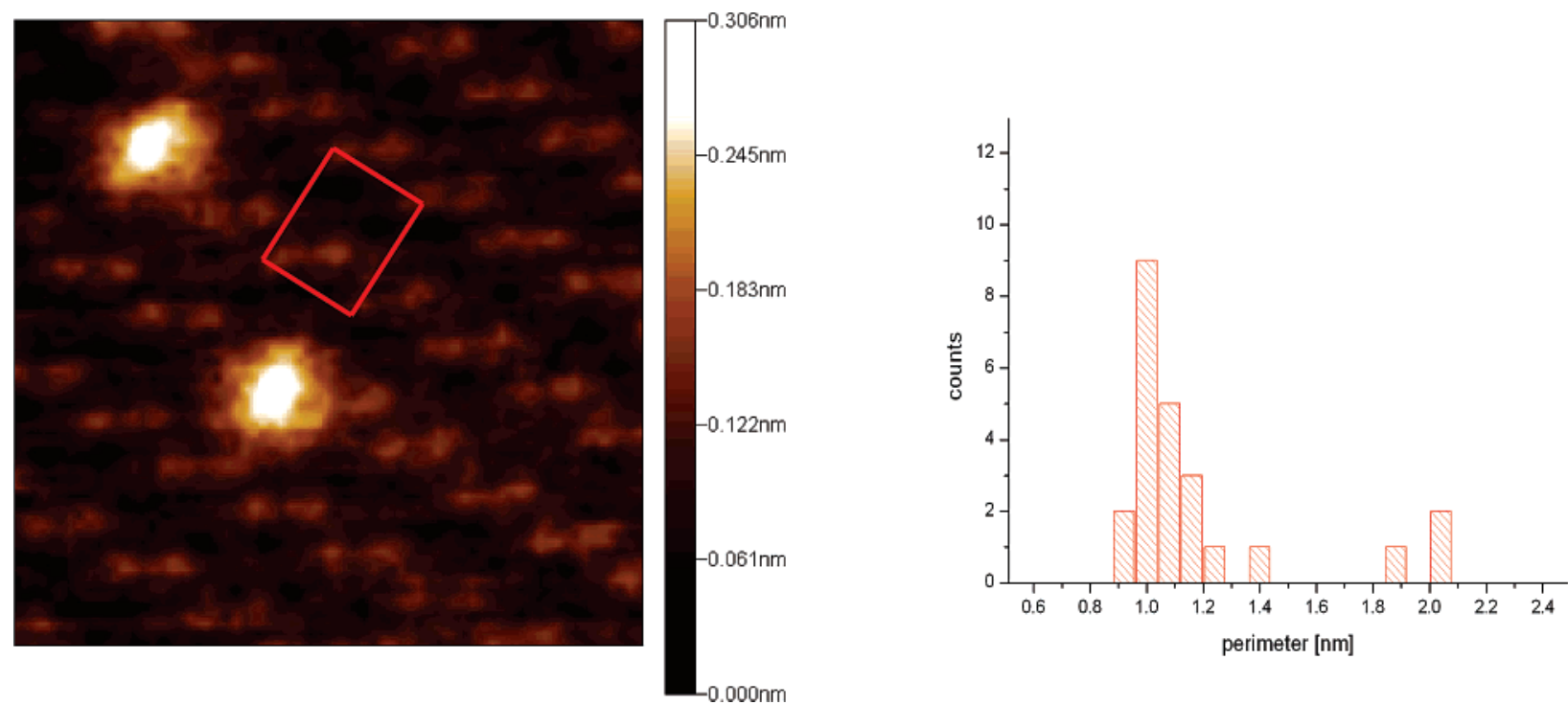

Figure 5. (a) Enlarged image of BP4 molecules inserted into a $\mathrm{C} 12$ domain $\left(6.7 \times 6.7 \mathrm{~nm}^{2}\right)$. The red box marks the $(3 \times 2 \sqrt{ } 3)$ unit cell of $\mathrm{C} 12$ (equivalent to the notation $\mathrm{c}(4 \times 2))$. (b) Distribution of the perimeter of the embedded molecules seen in Figure $2 \mathrm{~b}$ ). 
(a)

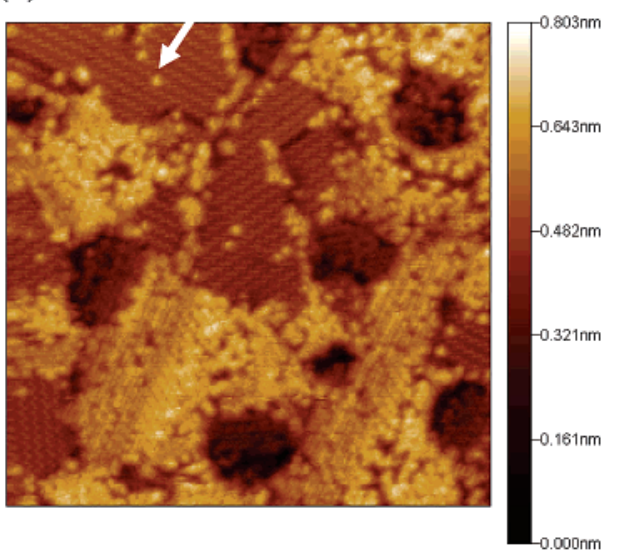

(b)

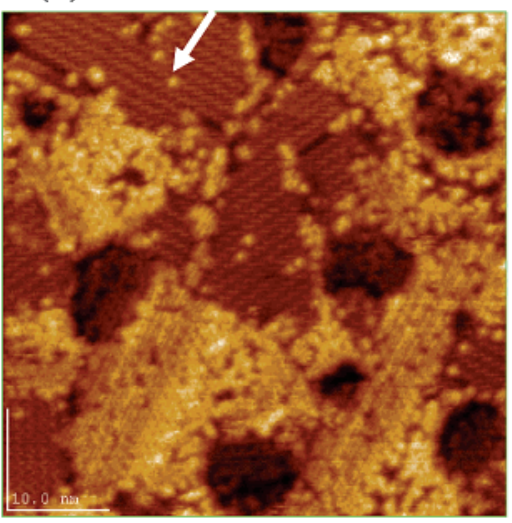

(c)

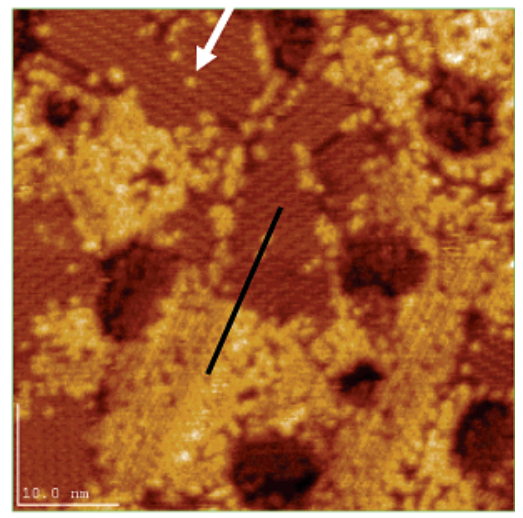

(d)

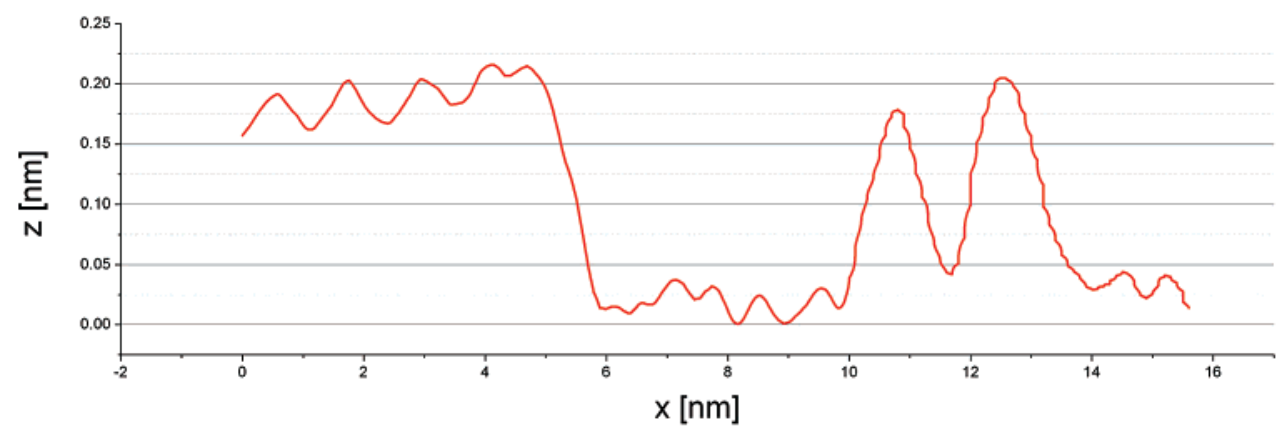

Figure 6. STM scan of the surface area (a) after $10 \mathrm{~min}$ and (b) after $16 \mathrm{~min}$; (c) $50 \times 50 \mathrm{~nm}^{2}$. No movement of BP4 molecules inserted into C12 domains is visible (see white arrow). The scale shown in part a is also valid for parts b and c. Part d shows a cross section along the black line in part c. The inserted single molecules have almost the same height as the BP4 domains.
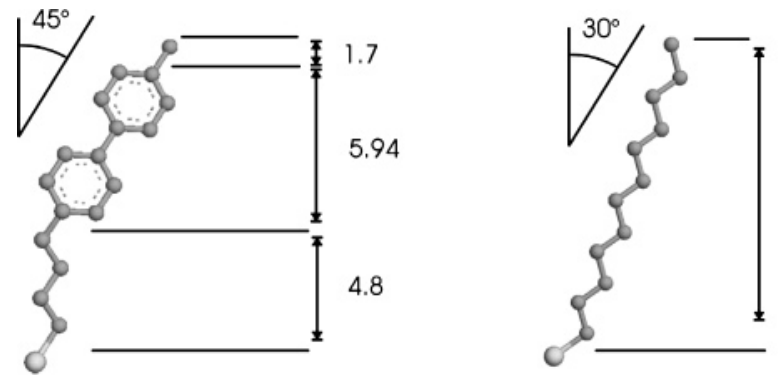

12.8

Figure 7. Orientation of BP4 and $\mathrm{C} 12$ in a full-coverage SAM. All lengths are given in angstroms.

difference of the BP4 and C12 domains. This kind of analysis is conducted for several scans, yielding the height differences as shown in Figure 4. The height difference slightly decreases from $0.148 \pm 0.045 \mathrm{~nm}$ without heating to $0.133 \pm 0.03 \mathrm{~nm}$ after $90 \mathrm{~min}$ and $0.11 \pm 0.021 \mathrm{~nm}$ after $3 \mathrm{~h} 45 \mathrm{~min}$ of annealing. The C12 domains already exhibited their final $\mathrm{c}(4 \times 2)$ structure before heating, making a strong rearrangement of the alkanes less probable. Therefore, most of the change in the height difference should result from an increase in order in the BP4 domains.

A closer look at Figure 2 a reveals another interesting property of the mixed C12/BP4 film. Some BP4 molecules are not incorporated into the film at defect sites such as domain boundaries and pits in the gold surface but into the middle of a C12 domain (white arrow in Figure 2a). However, this process has a much lower probability than the insertion at defect sites.

Figure 5 shows an enlarged image of an area in which BP4 molecules are inserted into a $\mathrm{C} 12$ domain. BP4 molecules appear as protrusions. Also visible is the $c(4 \times 2)$ structure of the underlying $\mathrm{C} 12$ structure, which provides a coordinate system to measure the lateral dimensions of the embedded molecules.
All protrusions appear to be smaller than one unit cell of the (3 $\times 2 \sqrt{ } 3$ ) structure (equivalent to the notation $c(4 \times 2)$, see red box in Figure 5), which consists of four C12 molecules per unit cell. This leads to the estimation that the protrusions consist of fewer than four BP4 molecules. Considering the relatively larger occupied area per molecule for BP4 compared to that for $\mathrm{C} 12$ and that the STM image is a convolution of the imperfect STM tip shape and surface geometry, the estimation of four molecules can be taken as the upper limit. Furthermore, the distribution of the perimeter of the protrusions is relatively sharp, as shown, for example, for the protrusions of Figure $2 \mathrm{~b}$ in Figure $5 \mathrm{~b}$. The distribution peaks at a perimeter of $1 \mathrm{~nm}$, but a few protrusions have a perimeter of approximately $2 \mathrm{~nm}$ (these protrusions appear elliptical; the length of the longer axis has been used). Therefore, we propose that the protrusions consist mainly of only a single BP4 molecule with some cases of two molecules per protrusion.

As already pointed out in the previous paragraph, the $c(4 \times$ 2) structure of $\mathrm{C} 12$ provides a coordinate system to determine the lateral size and position of the embedded BP4 molecules. This is especially advantageous for the study of diffusion processes of BP4 molecules within the $\mathrm{C} 12$ domains. Figure $6 \mathrm{a}-\mathrm{c}$ shows a scan of the same sample area and provides a comparison of this area after $10 \mathrm{~min}$ and after $16 \mathrm{~min}$ with respect to the initial state. For example, one inserted molecule is marked by a white arrow. It can be seen that the inserted molecules are fixed at their insertion place and do not diffuse. This observation is in agreement with the observation of Cygan et al. for more rigid conjugated oligomers ${ }^{24}$ and with the absence of phase segregation in SAMs of asymmetrical disulfides at room temperature. ${ }^{18,19}$ However, there is an ambiguity if the diffusion is significantly increased at higher temperatures. Whereas Ishida et al. ${ }^{35}$ observe phase separation in a SAM of an asymmetric disulfide with hydrocarbon and fluorocarbon chains, indicating cleavage of the $S-S$ bond 
and a diffusion coefficient on the order of $10^{-18} \mathrm{~m}^{2} \mathrm{~s}^{-1}$, Schönherr et al. observed no phase segregation in mixed alkyl fluoroalkyl disulfides ${ }^{20}$ either due to intact $\mathrm{S}-\mathrm{S}$ bonds or a very low diffusion coefficient. The setup presented here is highly convenient for solving this problem because the movement of BP4 molecules relative to the $\mathrm{C} 12$ structure can be precisely determined. Currently, temperature-controlled experiments are underway to determine the mobility of embedded molecules in a C12 domain exactly.

The mixed monolayer presented here is a system in which both different BP4 and C12 domains as well as single BP4 molecules embedded into $\mathrm{C} 12$ domains exist. These STM scans give us the unique ability to compare the structure of the BP4 domains, which should approach the structure of full-coverage BP4 monolayers, with that of single embedded molecules. As can be seen in the cross section in Figure 6d, the embedded molecules are almost the same height as the domains of BP4. This is an indication that the embedded molecules adopt the same conformation as the molecules in full-coverage SAMs.

As already mentioned in the Introduction, the height difference between the BP4 domains and the $\mathrm{C} 12$ layer can be interpreted in terms of different transconductances of these molecules ${ }^{37}$ For the following interpretation, the orientation of BP4 as shown in Figure 7 is assumed. The biphenyl group is tilted $45^{\circ}$ out of the surface normal, whereas the alkane chain has a rather upright orientation. ${ }^{36}$ Please note that the twist angle of the biphenyl group around its molecular axis is chosen arbitrarily. However, different twist angles would not change the results of the discussion below.

The conductance of a molecular wire can be expressed by the generally used exponential law ${ }^{37,48}$

$$
G=G_{0} \exp (-\beta d)
$$

where $G_{0}$ is the contact resistance, $\beta$ is the molecule-dependent decay constant, and $d$ is the molecular length.

Decay constants of alkanethiols $\beta_{\mathrm{CH}}$ have been measured by various methods, ${ }^{37,49-54}$ but less data exists for the decay constant of phenylens $\beta_{\mathrm{Ph}}{ }^{55-5855-59}$ Because BP4 consists of an alkane and a biphenyl part, we have split up the exponential term into an alkane-dependent and a phenyl-dependent part, leading to a two-layer model

$$
G_{\mathrm{BP} 4}=G_{0, \mathrm{BP} 4} \exp \left(-\beta_{\mathrm{CH}} d_{\mathrm{CH}}-\beta_{\mathrm{Ph}} d_{\mathrm{Ph}}\right)
$$

where $\beta_{\mathrm{CH}}$ and $\beta_{\mathrm{Ph}}$ are the decay constants of the alkane chain and the phenylen rings and $d_{\mathrm{CH}}$ and $d_{\mathrm{Ph}}$ are the lengths of the alkane chain and the biphenyl group.

(48) Magoga, M.; Joachim, C. Phys. Rev. B 1997, 56, 4722.

(49) Slowinski, K.; Fong, H. K. Y.; Majda, M. J. Am. Chem. Soc. 1999, 121 7257.

(50) Yasutake, Y.; Shi, Z.; Okazaki, T.; Shinohara, H.; Majama, Y. Nano Lett. $\mathbf{2 0 0 5}, 5,1057$.

(51) Wang, W.; Lee, T.; Reed, M. A. Phys. Rev. B 2003, 68, 035416.

(52) Wold, D. J.; Frisbie, C. D. J. Am. Chem. Soc. 2000, 122, 2970.

(53) Selzer, Y.; Salomon, A.; Cahen, D. J. Phys. Chem. B 2002, 106, 10432.

(54) Kaun, C.-C.; Guo, H. Nano Lett. 2003, 3, 1521.

(55) Wold, D. J.; Haag, R.; Rampi, M. A.; Frisbie, C. D. J. Phys. Chem. B 2002, 106, 2813.

(56) Holmlin, R. E.; Ismagilov, R. F.; Haag, R.; Mujica, V.; Ratner, M. A.; Rampi, M. A.; Whitesides, G. M. Angew. Chem., Int. Ed. 2001, 113, 2378.

(57) Wakamatsu, S.; Akiba, U.; Fujikira, M. Jpn. J. Appl. Phys. 2002, 41, 4998

(58) Wakamatsu, S.; Fujii, S.; Akiba, U.; Fujikira, M. Ultramicroscopy 2003 , 97, 19.

(59) The same approximation has also been applied by Moth-Poulsen et al. ${ }^{28}$ and Szuchmacher Blum et al. ${ }^{29}$ and for the homologous series of alkanethiols by Bumm et al. ${ }^{37}$
For a quantitative interpretation, we have to include the vacuum gap above $\mathrm{C} 12$ and BP4 domains $\left(G_{\mathrm{vac}}=G_{0, \mathrm{vac}} \exp \left(-\beta_{\mathrm{vac}} d_{\mathrm{vac}}\right)\right)$. In general, the parameters $G_{0 \text {,vac }}$ and $\beta_{\text {vac }}$ will be different above $\mathrm{C} 12$ and BP4 (leading to parameters $G_{0, \mathrm{vac}, \mathrm{C} 12}, G_{0 \text {,vac,BP} 4,} \beta_{\mathrm{vac}, \mathrm{C} 12}$, and $\left.\beta_{\mathrm{vac}, \mathrm{BP} 4}\right)$. However, following the experiments of Ishida et al., ${ }^{30}$ it has been assumed that $\beta_{\mathrm{vac}, \mathrm{C} 12}=\beta_{\mathrm{vac}, \mathrm{BP} 4}$. Ishida et al. have measured the increase in the local barrier height (LBH) between a nonanethiol (C9) SAM and 4-biphenylthiol and 4-biphenylmethanethiol. The latter molecule is especially similar to BP4. They observed that the LBH increases only by $0.24 \mathrm{eV}$ for 4-biphenylmethanethiol and by $0.84 \mathrm{eV}$ for 4-biphenylthiol. Because the LBH $\Phi$ is connected with the decay constant by $\Phi(\mathrm{eV})=0.952\left[\beta_{\mathrm{vac}}\left(\AA^{-1}\right)\right]^{2}$ and using the literature value $\beta_{\mathrm{vac}} \approx$ $2.3 \AA^{-1}, 37$ the error made by the approximation $\beta_{\mathrm{vac}, \mathrm{C} 12}=\beta_{\mathrm{vac}, \mathrm{BP} 4}$ is less than $8 \%$.

Including the vacuum gap, the tunnel conductance above $\mathrm{C} 12$ and BP4 domains can be rewritten as follows:

$$
\begin{gathered}
G_{\mathrm{BP} 4} G_{\mathrm{vac}, \mathrm{BP} 4}=G_{0, \mathrm{BP} 4} G_{0, \mathrm{vac}, \mathrm{BP} 4} \exp \left(-\beta_{\mathrm{CH}} d_{\mathrm{CH} \cdot \mathrm{BP} 4}-\beta_{\mathrm{Ph}} d_{\mathrm{Ph}}-\right. \\
\left.\beta_{\mathrm{vac}} d_{\mathrm{vac}, \mathrm{BP} 4}\right)(4) \\
G_{\mathrm{C} 12} G_{\mathrm{vac}, \mathrm{C} 12}=G_{0, \mathrm{C} 12} G_{0, \mathrm{vac}, \mathrm{C} 12} \exp \left(-\beta_{\mathrm{CH}} d_{\mathrm{CH}, \mathrm{C} 12}-\right. \\
\left.\beta_{\mathrm{vac}} d_{\mathrm{vac}, \mathrm{C} 12}\right)
\end{gathered}
$$

Measuring in constant current mode implies that $G_{\mathrm{BP} 4} G_{\mathrm{vac}, \mathrm{BP} 4}=$ $G_{\mathrm{C} 12} G_{\text {vac }, \mathrm{C} 12}$ leading to

$$
\beta_{\mathrm{Ph}}=\frac{\begin{array}{l}
\ln \left(G_{0, \mathrm{BP} 4} G_{0, \mathrm{vac}, \mathrm{BP} 4} / G_{0, \mathrm{C} 12} G_{0, \mathrm{vac}, \mathrm{C} 12}\right)+ \\
\beta_{\mathrm{CH}}\left(d_{\mathrm{CH}, \mathrm{C} 12}-d_{\mathrm{CH}, \mathrm{BP} 4}\right)-\beta_{\mathrm{vac}}\left(d_{\mathrm{vac}, \mathrm{BP} 4}-d_{\mathrm{vac}, \mathrm{C} 12}\right)
\end{array}}{d_{\mathrm{Ph}}}
$$

The difference in the vacuum gap $\Delta h_{\mathrm{gap}}=\left(d_{\mathrm{vac}, \mathrm{BP} 4}-d_{\mathrm{vac}, \mathrm{C} 12}\right)$ relates to the observed STM height $\Delta h_{\mathrm{STM}}$ and the physical height difference between BP4 and $\mathrm{C} 12 \Delta h_{\text {phys }}$ as follows:

$\Delta h_{\text {gap }}=\Delta h_{\text {phys }}+\Delta h_{\mathrm{STM}}=0.36 \AA+1.1 \AA=1.46 \AA$

BP4 and C12 have the same top and bottom groups and are scanned with the same tunneling tip. Therefore, it is assumed that $G_{0, \mathrm{C} 12} G_{0, \text { vac,C12 }} \approx G_{0, \mathrm{BP} 4} G_{0, \text { vac,BP4 }}$ (i.e., BP4 and $\mathrm{C} 12$ have the same contact resistances). Wold et al. have measured the contact resistances of alkanethiols and phenylenes and state that the contact resistances are indistinguishable within their experimental errors. ${ }^{55}$ This approximation probably works so well because only the logarithm of the ratio $G_{0, \mathrm{C} 12} G_{0, \mathrm{vac}, \mathrm{C} 12} /$ $G_{0, \mathrm{BP} 4} G_{0, \mathrm{vac}, \mathrm{BP} 4}$ enters the equation so that any error made by this approximation is small compared to the other summands. ${ }^{59}$

The literature values of $\beta_{\mathrm{CH}}$ are around unity (ranging from 0.7 to $1.2 \AA^{-137,49-54}$ ), so we have chosen a value of $\beta_{\mathrm{CH}}=1.0$ $\AA^{-1}$. The decay constant of vacuum was taken as $\beta_{\text {vac }} \approx 2.3$ $\AA^{-1} .37$ Using these values, it follows that $\beta_{\mathrm{Ph}}=0.5 \AA^{-1}$ for through-space tunneling (i.e., tunneling along the shortest connection between the tip and substrate, the lengths in Figure 7 have been used) and $\beta_{\mathrm{Ph}}=0.59 \AA^{-1}$ for through-bond tunneling (i.e., tunneling along the molecular backbone). These values agree well with the experiments of Wakamatsu et al. ${ }^{57,58}(0.53$ $\AA^{-1}$ for STM and $0.55 \AA^{-1}$ for AFM experiments) and are slightly lower than the values measured by Holmlin et al. ${ }^{56}$ who found a decay constant of $0.67 \AA^{-1}$ by mercury droplet measurements, which is slightly higher than the values measured by Wold et al. ${ }^{55}$ ( $0.42 \AA^{-1}$ for AFM experiments), implying that the rather rough approximations we applied seem to be justified. In this way, the apparent STM heights can be explained in terms of a higher tunneling conductance of BP4. Assuming through-bond 
or through-space tunneling leads to decay constants $\beta_{\mathrm{Ph}}$ within the variation of values found in the literature.

\section{Conclusions}

A method for depositing mixed SAMs consisting of BP4 and $\mathrm{C} 12$ is presented. The resulting film consists of different domains of BP4 and C12 molecules. Domain boundaries and pits in the gold surface act as nucleation points for BP4 domains, whereas the nucleation at pits seems to be more effective. Annealing of the film at $64{ }^{\circ} \mathrm{C}$ results in a slight decrease of the height of BP4 domains above $\mathrm{C} 12$.

For the presented experimental conditions, some BP4 molecules are found to be inserted into the middle of a $\mathrm{C} 12$ domain. The size and position can be determined by using the underlying c(4 $\times 2)$ structure of the alkanethiols as the coordinate system. Thereby, it is shown that the protrusions in the C12 domains contain fewer than four molecules and are most probably due to single embedded BP4 molecules. Furthermore, these molecules are fixed in the $\mathrm{C} 12$ structure and do not migrate at room temperature. Comparing the height of the embedded molecule with the height of the BP4 domains, we propose that the embedded single BP4 molecules have the same conformation as in the full-coverage phase.

The height difference of the domains of BP4 compared to the C12 SAM is explained in terms of a higher tunneling conductance of BP4. A simple two-layer model is proposed by which the current transport through the BP4 group is discussed and the decay constant $\beta_{\mathrm{Ph}}$ is deduced.

Acknowledgment. We thank J. Kiesgen, A. Basler, and B. Hahn for preparing the BP4, H. Haselier for the gold substrates, and K. Szot for his assistance.

LA052791U 